\title{
Cesure e continuità nelle autobiografie 'armate' in Italia
}

Lorenzo De Sabbata $^{1}$

This article analyses two autobiographical writings produced by ex-militants of left-wing armed organisations active in Italy during the 'Years of Lead'. These writings will be considered contributions to a widespread, connected process of public 'memorialisation', which they negotiate with other historical accounts. Their goal is to (re)produce existential, historical, and political representations of the authors' individual trajectories as well as those of their organisations. The aim of the article is to examine how the authors selected, portrayed, and contextualised the ruptures and continuities in their individual trajectories, focusing especially on their estrangement from the armed struggle. Although in both cases estrangement is represented as a process, the analysis is going to focus on the different events that articulate it as well as on how these events are arranged on a more public or private level.

Questo articolo nasce da una lettura problematizzata di due scritti autobiografici prodotti da ex-militanti di organizzazioni armate di sinistra attive in Italia durante gli 'anni di piombo'. Attraverso questi dispositivi letterari, gli ex-militanti intervengono in un processo di memorializzazione pubblica stratificato e corale. Prendendo posizione in un 'dialogo' con altre raffigurazioni - memorialistiche, giornalistiche e scientifiche - questi ex militanti (ri)producono rappresentazioni esistenziali, storiche e politiche della propria traiettoria personale e di quella dell'organizzazione di appartenenza.

L'obiettivo di questo articolo è di analizzare come i diversi autori abbiano individuato, narrativamente svelato e contestualizzato i punti di rottura e di continuità delle loro esperienze, concentrandosi in particolare sull'allontanamento dalla strategia armata. Se, in entrambi i casi, questo è descritto come un processo, l'analisi si concentra sulle diverse scelte evenemenziali che lo scandiscono temporalmente e sulla loro articolazione sul piano pubblico e personale.

\footnotetext{
${ }^{1}$ È dottorando presso il Centre de Recherches Historiques dell'École des Hautes Études en Sciences Sociales di Parigi.
} 


\section{Dalla parola delle armi alle armi della parola}

Dalla metà degli anni '80 a oggi, il paesaggio editoriale italiano si è progressivamente popolato di 'memorie armate', narrazioni autobiografiche prodotte da ex militanti di organizzazioni armate di estrema sinistra attive in Italia dall'inizio degli anni ' 70 , anni che si caratterizzano per l'ampiezza dei fenomeni legati all'impiego della violenza a fini politici. Il Paese, infatti, è funestato dalle stragi - gli attentati dinamitardi di matrice neofascista che, tra il 1969 e il 1982, costano la vita a 135 persone -, dai progetti e tentativi di golpe e dai frequenti scontri di strada che oppongono militanti di diverse estrazioni politiche e forze di polizia ${ }^{3}$. L'attività delle organizzazioni armate di estrema sinistra raggiunge il suo culmine tra il $1978 \mathrm{e}$ il 1982. Negli stessi anni, le pressioni delle forze dell'ordine aumentano di vigore : la creazione delle carceri speciali di massima sicurezza destinate ai militanti si accompagna all'approvazione, nel 1980, della cosiddetta 'legge Cossiga' che introduce sconti di pena per i collaboratori di giustizia e aggravanti specifiche per i condannati per reati con finalità terroristiche.

L'approvazione della legge fa seguito ai primi casi di pentitismo e sancisce la collaborazione con le forze dell'ordine di alcuni militanti in cambio di cospicui sconti di pena: nessun intimo pentimento personale è però richiesto e la legge di limita a istituzionalizzare il do ut des tra Stato e collaboratore.

Se la diffusione del pentitismo - nell'agosto 1982, 300 detenuti hanno usufruito della legge ${ }^{4}-$ accelera la frammentazione e l'estinzione delle organizzazioni armate all'esterno delle carceri, all'interno contribuisce a creare un clima di sospetto e controllo tra gli stessi militanti - la cosiddetta 'guerra agli infami' - che porta alla morte o al suicidio di alcuni 'traditori' o dei loro familiari ${ }^{5}$. Questa situazione spinge gruppi di 'prigionieri politici' a riconoscersi invece in un'idea di 'desistenza' : ammissione delle proprie responsabilità penali, distacco dalla violenza come strategia politica e rifiuto della collaborazione intesa come denuncia degli ormai ex complici. Il riconoscimento della posizione giuridica dissociativa - sancita legalmente, nel febbraio 1987, con il contributo di una parte dei 'detenuti politici' - è stato particolarmente

\footnotetext{
2 Emmanuel, BETTA, « Memorie in conflitto. Autobiografie della lotta armata », Contemporanea. Rivista di storia dell'800 e del '900, v. 4, 2009, pp. 673-702.

${ }^{3}$ Mirco DONDI, L'eco del boato : storia della strategia della tensione (1969-1974), Roma-Bari, Laterza, 2015 ; Guido CRAINZ, Il paese mancato : dal miracolo economico agli anni ottanta, Roma, Donzelli, 2003.

${ }^{4}$ Monica GALFRÉ, La guerra è finita. L'Italia e l'uscita dal terrorismo (1980-1987), Roma-Bari, Laterza, 2014, p. 83. Anna CENTO BULL e Philip COOKE, Ending terrorism in Italy, London-New York, Routledge, 2013.

${ }^{5}$ Maria Rita PRETTE (a cura di), Sguardi ritrovati, Roma, Sensibili alle foglie, 1995, pp. 310-313.
} 
tormentato e ha dato luogo a una varietà di esiti disomogenei e contrapposizioni che sono indicativi della difficoltà di considerare la dissociazione, così come il pentitismo, una categoria stagna, monolitica e coerente. In sostanza, la legge annulla gli effetti delle aggravanti per terrorismo, permettendo a chi ne beneficia di accedere a permessi e misure di detenzione alternativi. Una stima calcola che ne avrebbero beneficiato 3013 condannati ${ }^{6}$. Il 1987 è anche il momento in cui una parte dei cosiddetti 'irriducibili' storici - come Renato Curcio - senza dissociarsi né pentirsi, dichiara pubblicamente l'inattualità della lotta armata.

In questi stessi anni alcuni militanti, con il distacco dalla pratica armata, cominciano a produrre e pubblicare le loro memorie autobiografiche. La maggioranza di testi pubblicati proviene dall'area delle Brigate Rosse (BR), comprendente anche le organizzazioni che hanno fatto seguito alla frammentazione del gruppo storico. L'egemonia brigatista è indicativa di un'inclinazione mediatica e del mercato editoriale - e, di conseguenza, dei fruitori - verso l'esperienza di questa organizzazione : il sapere pubblico sulla lotta armata di sinistra sembra identificarsi quasi totalmente con la storia delle BR e l'affaire Moro diventare la vera 'metonimia degli anni di piombo', anche a seguito delle più recenti politiche memoriali. Nel corso degli anni '90, il ruolo giocato dalle memorie armate nella rappresentazione pubblica degli anni '70 trasforma il loro valore originario di 'testimonianza' in 'competenza' ${ }^{7}$. Le esperienze estreme della militanza, attestate dalle narrazioni autobiografiche, diventano una expertise grazie alla quale alcuni militanti sono sollecitati ad esprimersi pubblicamente su questioni di attualità politica. Alcuni di questi, inoltre, uniscono l'autorità della parola 'di chi c'era' a una metodologia pseudo-scientifica per sostenere ricostruzioni di stampo dietrologico volte a indagare i presunti misteri nella storia della sinistra armata in Italia ${ }^{8}$.

Questa centralità memoriale della 'parola armata' viene rimessa in questione col riapparire della sigla BR, con gli omicidi di Massimo D'Antona e Marco Biagi : le critiche verso la sovraesposizione mediatica degli 'ex terroristi' coinvolgono presto il mondo della politica nella ingiunzione al silenzio rivolta agli ex militanti armati ${ }^{9}$. Anche da un punto di vista editoriale,

\footnotetext{
${ }^{6}$ M. GALFRÉ, La guerra è finita, op. cit., pp. 99-115.

${ }^{7}$ Cf. E. BETTA, Memorie in conflitto, op.cit.

${ }^{8}$ Giovanni FASANELLA e Alberto FRANCESCHINI, Che cosa sono le BR. Le radici, la nascita, la storia, il presente, Milano, Rizzoli, 2004.

9 Giorgio NAPOLITANO, «Un omaggio », in Per le vittime del terrorismo nell'Italia repubblicana : in occasione del Giorno della memoria dedicato alle vittime del terrorismo e delle stragi di tale matrice, Roma, Istituto Poligrafico e Zecca dello Stato, 2008.
} 
con l'inizio degli anni 2000 le memorie armate trovano sempre meno interesse da parte delle case editrici di massa dove vengono 'sostituite' dalla memorialistica dei familiari delle vittime ${ }^{10}$.

In una prospettiva pragmatica, queste scritture di sé possono essere intese come prese di posizione nella sfera pubblica italiana il cui obiettivo è orientare o contrastare altri discorsi mediatici, riflessioni accademiche e politiche giudiziarie o memoriali sugli anni di piombo ${ }^{11}$. Adottando una prospettiva microstorica e biografica ${ }^{12}$, questo articolo si propone di analizzare due di queste narrazioni autobiografiche - Io l'infame di Patrizio Peci e Miccia corta di Sergio Segio $^{13}$ - al fine di indagare le maniere in cui, attraverso le rispettive « prese della parola scritta ${ }^{14}$, gli autori hanno individuato e narrativamente svelato i punti di rottura e di continuità delle loro esperienze di militanza e di post-militanza, operando nel presente della scrittura una riconfigurazione della propria traiettoria rispetto all'esperienza della violenza armata.

Io, l'infame è il primo testo di questo tipo a essere pubblicato da una casa editrice nazionale, nel 1983 e, dopo varie ristampe, uscirà dai cataloghi per poi rientrarvi con una nuova edizione nel 2008. Miccia corta viene invece pubblicato nel 2005, in coincidenza con la liberazione definitiva del suo autore. Da un punto di vista formale, questi testi mostrano alcune delle diverse articolazioni possibili delle memorie armate. Se quella di Segio è una autobiografia 'classica' in cui protagonista, narratore ed autore coincidono, Io, l'infame è una " eterobiografia ${ }^{15}$ : accreditato come curatore del volume, Bruno Guerri né è in realtà l'autore materiale, avendo

\footnotetext{
${ }^{10}$ Vedi : Mario CALABRESI, Spingendo la notte più in là : storia della mia famiglia e di altre vittime del terrorismo, Milano, Mondadori, 2007 ; Benedetta TOBAGI, Come mi batte forte il tuo cuore : storia di mio padre, Torino, Einaudi, 2009.

${ }^{11}$ L'espressione periodizzante 'anni di piombo' rimane molto discussa. Qui è utilizzata per definire in modo generico il periodo di attività delle organizzazioni armate. Vedi : Carmela LETTIERI, L'Italie et ses Années de plomb. Usages sociaux et significations politiques d'une dénomination temporelle, Mots. Les langages du politique [in linea], n. 87, 2008, URL : http://mots.revues.org/12032 (consultato il 10 ottobre 2016).

${ }^{12}$ Giovanni LEVI, Les usages de la biographie, Annales E.S.C., v. 44 n.6, 1989, pp. 1325-1336 ; Sabina LORIGA, Le Petit x. De la biographie à l'histoire, Paris, Seuil, 2010.

${ }^{13}$ Patrizio PECI, Io, l'infame (a cura di Giordano Bruni Guerri), Milano, Mondadori, 1983 ; Sergio SEGIO, Miccia Corta. Una storia di Prima Linea, Roma, Deriveapprodi, 2005.

${ }^{14}$ Philippe ARTIÈRES, Le livre des vies coupables : autobiographies de criminels (1896-1909), Paris, Albin Michel, 2000.

${ }^{15}$ Philippe LEJEUNE, « L'autobiographie de ceux qui n'écrivent pas. Qui est l'auteur ? », in id., Je est un autre, Paris, Seuil, 1980, pp. 223-252.
} 
trasposto una lunga intervista con Peci in un testo alla prima persona singolare di cui l'ex militante è protagonista ${ }^{16}$.

A questo punto, è importante ricostruire più nel dettaglio le traiettorie di militanza dei due autori. Patrizio Peci cresce in provincia e si avvicina alla politica solo agli inizi degli anni ' 70 partecipando alle attività organizzate in zona dalla sinistra extraparlamentare. La sua militanza cambia passo con l'avvicinamento alle BR, nel 1976, e il successivo trasferimento a Milano e poi a Torino, dove diventa uno dei dirigenti dell'organizzazione fino al suo arresto, il 19 febbraio del 1980. Durante la militanza, Peci partecipa in prima persona a varie azioni tra cui alcune gambizzazioni intimidatorie e l'attentato omicida al giornalista Carlo Casalegno. A circa un mese dal suo arresto, decide di collaborare col nucleo speciale anti-terrorismo dei Carabinieri diretto del generale Alberto Dalla Chiesa : diventa così il primo grande pentito della storia delle $\mathrm{BR}$, e la sua collaborazione renderà possibile l'arresto di molti militanti e la scoperta di vari covi dell'organizzazione. Nel 1981, le BR-Partito Guerriglia - un'altra delle fazioni in cui si erano frammentate dalle BR 'originarie' - sequestrano il fratello di Peci, indicato come simpatizzante dell'organizzazione e sospetto collaboratore. Come ritorsione per la sua delazione e quella del fratello, e per dare un esempio, Roberto Peci viene sottoposto a un 'processo popolare' filmato che termina nella sua condanna a morte. Dopo il suo pentimento, Peci viene scarcerato - dopo circa cinque anni di prigione - e acquisisce una nuova identità sotto la protezione dei carabinieri.

Sergio Segio cresce a Sesto San Giovanni, la 'Stalingrado d'Italia', e si avvicina alla politica attraverso la militanza in Lotta Continua nella prima metà degli anni '70. Nel 1976-1977 contribuisce alla fondazione dell'organizzazione armata Prima Linea (PL). Durante la sua militanza in PL, Sergio Segio partecipa in prima persona all'omicidio dei magistrati Emilio Alessandrini e Guido Galli e del fiancheggiatore 'pentito' William Vaccher. Arrestato nel 1983, dopo alcuni mesi di detenzione, dichiara assieme alla quasi totalità dei membri di PL lo scioglimento dell'organizzazione diventando ben presto uno dei volti più conosciuti del movimento della dissociazione. Nel 1987, usufruisce della legge omonima e accede progressivamente alle misure di detenzione alternative iniziando la sua attività di operatore sociale con l'associazione torinese Gruppo Abele.

\footnotetext{
${ }^{16}$ La questione della co-autorialità di queste e di molte altre memorie armate rimane fondamentalmente inesplorata e di difficile investigazione documentaria. Le geografie formali di queste autorialità condivise, infatti, restano decrittabili solo parzialmente grazie alle indicazioni fornite dagli stessi autori nei paratesti.
} 


\section{2. lo, l'infame : l'evento biografico come disvelamento della realtà}

La pubblicazione di Io, l'infame ha una particolare valenza politica : si tratta del racconto autobiografico del primo pentito delle BR che prende la parola pubblicamente, fuori dalle aule giudiziarie, rivolgendosi a tutto quel mondo sociale che si sente e viene presentato dai media come in guerra con le organizzazioni armate. È una delle prime opportunità per un pubblico più ampio di entrare in contatto con il 'mondo terrorista' attraverso lo sguardo di un protagonista ormai pentito che ha così preso distanza sia dal suo passato criminale che dal presente armato ancora incarnato dai terroristi 'irriducibili'.

L'obiettivo principale del libro è eminentemente pedagogico : l'autore vuole partecipare attivamente allo smantellamento dell'immagine pubblica delle BR raccontando la « vita quotidiana del 'povero brigatista' per togliere qualsiasi alone mitico e leggendario all'Organizzazione ${ }^{17}$. A questo fine, nel testo vengono utilizzate due tecniche narrative convergenti : l'insistenza sull'aspetto comico-grottesco nella descrizione di personaggi e situazioni dell'universo brigatista e il racconto dettagliato delle modalità e degli effetti della violenza sulle vittime degli attentati. Usando in modo sequenziale il registro della derisione e quello della disumanizzazione, Peci costruisce una differenza tra se stesso ed i suoi ex compagni. Le BR diventano una « armata Brancaleone » di personaggi ridicoli e patetici che intraprendono delle azioni al di fuori della loro portata il cui successo deriva solo dalla fortuna e dall'effetto sorpresa. I personaggi principali di questi episodi - rivoluzionari raffazzonati e pasticcioni - sono i membri della colonna torinese, come Maria Rosaria Roppoli, ex fidanzata di Peci. Nel descriverla, egli fa costantemente riferimento al suo aspetto fisico sgradevole - da cui il suo soprannome 'Rospoli' - : « Racchia di viso, bassa, grassa, culo largo e basso » e ancora psicologicamente, militarmente e politicamente «un disastro $»^{18}$. La commedia dileggiante è interrotta solo dalle descrizioni degli attentati, frammenti narrativi di incredibile durezza e realismo da "manuale tecnico del brigatista ${ }^{19}$, che tradiscono i processi di disumanizzazione che rendono possibili queste azioni e il loro successivo racconto. Ecco un estratto che istruisce sulla gambizzazione : "Quando il bersaglio è in piedi, per prima cosa bisogna sparargli un paio

\footnotetext{
${ }^{17}$ P. PECI, Io, l'infame, op. cit., p. 114.

${ }^{18}$ Ibid., pp. 96-98.

${ }^{19}$ Giuliano TABACCO, Libri di piombo. Memorialistica e narrativa della lotta armata in Italia, Milano, Bietti, 2010 , p. 107.
} 
di colpi a caso, nelle gambe, perché cada. Si aspetta che cada perché se si spara mentre cade ci sono buone possibilità di ammazzarlo. [...] a quel punto si spara il resto dei colpi : tre, cinque, sei, dipende dal calibro, dalla precisione e dalla rabbia. È importante sparare tanti colpi per essere sicuri di colpire l'osso, anche se dal punto di vista politico l'azione può considerarsi riuscita con una ferita qualsiasi $»^{20}$. A differenza di altri scritti autobiografici, Io, l'infame elude qualsiasi tipo di riflessione politica sull'esperienza armata, aspetto che unito alla violenza esplicitata - solitamente grande rimosso narrativo di questi testi - rende l'opera senza equivalenti nel suo genere.

Il racconto della militanza di Peci non restituisce al lettore un protagonista dinamico, bensì un personaggio statico coinvolto in una serie di scene 'atemporali' che sembrano ripetersi senza soluzione di continuità. Nonostante sia scandito da riferimenti cronologici puntuali, Io, l'infame suggerisce che la militanza e la clandestinità siano un periodo di sospensione temporale in cui $\mathrm{i}$ brigatisti sono completamente assorbiti dal presente e perdono ogni capacità - soprattutto morale e politica - di concepire temporalità distinte da quelle che vivono. Anche i momenti di crisi sono ricondotti a questo schema tanto da sovrapporsi alla militanza stessa : "Certe crisi durano mesi, anni. Uno ci si arrovella, ci si rotola dentro, ci combatte, ora le vince, ora le perde, ma va

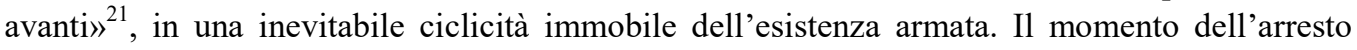
introduce una cesura nel presente e produce un orizzonte di futuro differente e contraddistinto dalla carcerazione. La stasi latente, la cosiddetta normalità, precipita dunque a causa dell'incontro con lo Stato : non più entità teorica, lo Stato acquisisce spazialità concreta nei muri della prigione e 'umanità' tramite i suoi rappresentanti. L'arresto è quindi la morte - « la mia vita era finita $\gg$ dice l'autore ${ }^{22}$ - che prelude alla rinascita.

La breve parentesi di Peci 'morto vivente' è un racconto di spazi angusti ed ostili - il carcere abitati da personaggi benevoli, i poliziotti. I pochi accenni alla violenza fisica sono essenzialmente bonari, nessun riferimento ai pestaggi che invece costellano la maggior parte delle altre memorie brigatiste : « io non sono mai stato toccato, né in caserma né in carcere [...]. Certo, qualche schiaffo può volare, così, alla buona, nell'agitazione $»^{23}$.

Il passaggio dalla morte alla nuova vita è scandito dagli incontri col generale Dalla Chiesa e il magistrato Caselli : rispetto alla bassezza e alla mediocrità dei brigatisti, questi personaggi sono

${ }^{20}$ P. PECI, Io, l'infame, op. cit., p. 127.

${ }^{21}$ Ibid., p. 173.

${ }^{22}$ Ibid., p. 183.

${ }^{23}$ Ibid., p. 180. 
presentati come uomini eccezionali. Questo è il momento in cui, agli occhi di Peci, si palesa la sproporzione di forza tra Stato e BR : la lotta armata è paragonata al « cercare di abbattere un elefante a colpi di spillo. Riuscivamo solo a rompergli i coglioni » e l'autore comincia così a percepire l'inevitabilità della fine : « eravamo sconfitti, militarmente e politicamente $»^{24}$. Peci quindi si 'dissocia', esce dall'organizzazione e comincia a collaborare. La strage di via Fracchia, l'uccisione di quattro brigatisti durante l'irruzione dei carabinieri nel covo loro indicato da Peci, fa però scattare la molla di quello che lui stesso chiama 'il vero pentimento' che si sviluppa nell'incontro col magistrato Caselli. Anche in questo caso, l'autore insiste sulla statura del personaggio - « uomo preparatissimo, vero professionista, di grande umanità [...] mi fece una grandissima impressione, anche più di Dalla Chiesa »- che contrasta con la bassezza degli ex compagni ${ }^{25}$. Questo incontro è l'acme narrativo che rappresenta la rinascita di Peci pentito che da terrorista diventa esempio. L'autore insiste molto su questo cambiamento, incorporando nel racconto di questo momento una serie di aspetti che richiamano la (ri)nascita : un travaglio che dura « due giorni, praticamente senza soste » con Dalla Chiesa che «passeggiava in corridoio come quei papà delle barzellette mentre aspettano il primo figlio $»^{26}$. L'arresto e il pentimento diventano quindi gradi di un vero e proprio processo di disvelamento della realtà. La metafora della presa di visione è fondamentale : « vivendo all'interno dell'organizzazione riesci a vedere solo lei e anche un fuscello, messo davanti agli occhi, ti sembra un pioppo. In carcere vedi solo lo stato $»^{27}$. La stessa idea è ribadita dal co-autore nella sua prefazione : « Come in un fenomeno di presbitismo, il terrorista non riesce a vedere la debolezza e la miseria dell'organizzazione finché ne è all'interno. Una volta catturato, la logica brutale e perversa del terrorismo appare in tutta la sua inconsistenza $»^{28}$.

Quando Peci rincontra gli ex compagni nel 1983, al processo nel quale testimonia contro di loro, il disvelamento viene 'confermato' dalla tragedia della morte del fratello e il registro di demonizzazione e disumanizzazione diventa predominante : i brigatisti sono ormai « belve e bestie e vederli in gabbia non mi fa nessuna impressione. Sono bestie per la ferocia con la quale non vogliono riconoscere che hanno perso e continuano a propagandare morte anche se sanno

\footnotetext{
${ }^{24}$ Ibid., pp. 193-194.

${ }^{25}$ Ibid., p. 198.

${ }^{26}$ Ibidem.

${ }^{27}$ Ibid., p. 193.

${ }^{28}$ Ibid., pp. 7-8.
} 
che la lotta armata non ha sbocchi $»^{29}$. Questi due ultimi aspetti, il riconoscimento della sconfitta e la fine dell'esercizio insensato della violenza, sono quello che ormai differenzia Peci dai suoi ex compagni. Malgrado egli si definisca un "'mostro' rimbalzato da una parte all'altra della barricata $»^{30}$, la virgolettatura del termine ne riduce la portata, poiché, in fondo, se Peci è stato carnefice in passato, ora è una vittima in pericolo, che ha sofferto l'uccisione del fratello e sperimenta il desiderio di vendetta della compagine brigatista. Il protagonista si rassegna alla sensazione di «non appartenere a nessun mondo », come se quel rimbalzo da una parte all'altra sia stato imperfetto, bloccato della società che lo perdona solo ufficialmente perché in realtà continua a considerarlo « un pazzo, un alieno o, peggio, un opportunista $»^{31}$.

\section{Sergio Segio : l'evento come Miccia corta della malinconia}

Un primo aspetto da sottolineare affrontando l'analisi del libro di Sergio Segio è la diversa ricezione di cui ha beneficiato presso il pubblico italiano : all'ultimo censimento, le copie vendute da Segio sono solamente 2.000, contro le circa 30.000 di Peci ${ }^{32}$. Non si tratta, come si potrebbe pensare, di una semplice questione di longevità : il libro di Peci, infatti, ha goduto di ottime vendite sin dalla sua pubblicazione, come certificano le immediate ristampe che hanno seguito la prima. La sorte editoriale del testo di Segio è particolarmente importante perché conferma e amplifica la sensazione di marginalità memoriale che ne è la motivazione principale. L'autore, infatti, percepisce la sua storia e quella della sua organizzazione come distorte e cancellate dalla memoria pubblica e dalla riflessione storica sugli anni di piombo in Italia. L'obiettivo esplicito del libro, quindi, è di reintegrarvele, ricostruendole tramite un evento, l'assalto al carcere di Rovigo, un episodio di grande importanza « sul piano della vicenda personale » di Segio e, al contempo, « fortemente rappresentativo del culmine e della fine di una storia e [...] di un'epoca, quella della rivolta armata $»^{33}$. L'introduzione esplicita lo sforzo di raccontare questo evento « con gli occhi, i riferimenti, i sentimenti, i linguaggi e le categorie di allora $»^{34}$, senza coscienza del dopo. La struttura dell'opera è pensata per sostenere questo

\footnotetext{
${ }^{29}$ Ibid., pp. 11-12.

${ }^{30}$ Ibid., p. 12.

${ }^{31}$ Ibidem.

${ }^{32}$ Cf., E. BETTA, Memorie in conflitto, op . cit.

${ }^{33}$ S. SEGIO, Miccia Corta, op. cit., p. 7.

${ }^{34}$ Ibidem.
} 
progetto che vorrebbe mettere in scena narrativamente l'annullamento di uno dei tratti marcanti l'esperienza autobiografica : la tensione tra passato dell'esperienza e presente della scrittura. Oltre al frammento autobiografico su Rovigo, il libro si compone di una lunga introduzione critica e di una appendice composta da testi diversi, lettere di ex militanti e articoli di giornale. Questo montaggio letterario permette di percepire in trasparenza i vari livelli di autorialità assunti da Segio : autobiografo e protagonista del suo racconto, firma l'introduzione - scritto analitico che serve a incorniciare il frammento nel contesto biografico, storico e di pubblicazione - ed infine curatore che organizza i testi che compongono l'appendice e di cui a volte è anche autore. È questa stessa struttura che permette la trasgressione delle regole narrative enunciate nell'introduzione ed è funzionale all'obiettivo del testo : situare la biografia dell'autore e l'esperienza di PL in una storia più vasta, quella della sinistra di classe, al fine di ridonare loro una legittimità e una cittadinanza politica.

Se il 15 gennaio 1982 - giorno dell'assalto - è il 'presente' del racconto, la contestualizzazione segue due direzioni : nel passato, per spiegare la scelta e le vicende che hanno portato fino a questo presente - l'« a monte » dell'evento - e nel futuro - per rivelare al lettore il suo $«$ a valle ${ }^{35}$ - fino al vero presente nascosto della scrittura.

Il viaggio nel passato ha luogo nell'introduzione e attraverso flashback pedagogici : appoggiandosi a citazioni di saggi scientifici, l'autore ricostruisce l'atmosfera degli anni '70 insistendo sulla violenza neofascista e poliziesca e sulla sensazione, molto diffusa a sinistra, della contiguità tra istituzioni democratiche e poteri eversivi. Nel racconto, questa sensazione diviene anacronisticamente certezza, grazie alla proiezione nel passato di una ricostruzione storica possibile solo a posteriori che ha l'effetto di rendere più legittimo, agli occhi del lettore, il passaggio dalla forza della ragione alle ragioni della forza. In questo senso, anche il tentativo dichiarato di rinunciare al senno di poi, sin da subito difficilmente percorribile, è un modo di insistere sul patto autobiografico e, conseguentemente, sull'effetto di veridicità storica della ricostruzione.

La storia di Segio e di PL è raccontata attraverso un'oscillazione incessante tra prima persona del singolare e del plurale, con la seconda che egemonizza i momenti di flashback e alcune parti dell'introduzione, dando l'impressione che l'autore sia fisicamente presente a tutte le tragedie

\footnotetext{
${ }^{35}$ Marie-Vic OZOUF-MARIGNIER e Nicolas VERDIER, « L'événement : un objet historique à emprunter », Éspace géographique, 2000, v. 29 n³, p. 219. Vedi : Reinhary KOSELLECK, Le Futur passé. Contribution à la sémantique des temps historiques, Paris, EHESS, 1990.
} 
individuali e collettive che hanno insanguinato gli anni ' $70^{36}$. La violenza, quindi, prima di essere scelta, è subita e le ragioni personali si dissolvono nei traumi del gruppo sociale e culturale di elezione. In questo racconto di sé politico e collettivo, il corpo individuale dell'autore-narratore-personaggio è rimpiazzato da un corpo collettivo e, seguendo l'intuizione di Maurice Bloch, sembra non esservi alcuna differenza tra memoria autobiografica e memoria semantica, tra ricordi vissuti e ricordi sentiti e poi evocati ${ }^{37}$.

L'evento Rovigo è centrale anche nell'economia del racconto della fine della militanza di Segio. Nel primo capitolo, il cui titolo Inverno dell'81 fornisce l'inquadratura cronologica, Segio è già fuoriuscito dall'organizzazione da qualche mese. «La militanza è sempre più degenerata $\mathrm{e}$ si è disumanizzata nella logica militare » scrive, " considero la guerra finita $»^{38}$. Attraverso flashback successivi viene ricostruita la progressione narrativa di questo distacco che trova le sue radici già nel primo omicidio politico effettuato da PL, nell'aprile 1976, fino a quello di William Vaccher, azione che segnala a Segio che « l'assalto al cielo è fallito » ${ }^{39}$. L'abbandono delle armi, però, non è pensabile : troppi latitanti e prigionieri, impossibile abbandonarli, sciogliere quel corpo collettivo con una soluzione individuale, soprattutto dopo l'arresto della sua compagna, Susanna. Tutto quindi converge verso l'evento Rovigo il cui esito effimero sembra rappresentare la soluzione a una crisi personale e sentimentale - la liberazione di Susanna - e aprire una nuova fase in cui la lotta armata sia «strumento di liberazione e di trattativa politica con lo stato ${ }^{40}$ per una amnistia generalizzata.

Tuttavia, il frammento biografico si conclude con un presagio di sventura : " una sensazione di inutilità della lotta, una certezza che l'amore, la speranza, la gioia, tutto sia già dietro alle spalle» ${ }^{41}$. Attraverso questa conoscenza anacronistica del futuro l'autore infonde nel testo una forte malinconia, una nostalgia di qualcosa che non si è veramente vissuto. L'esperienza mancata, nel racconto, è quella della scelta : forzato a ricorrere alle armi ma, allo stesso tempo, destinato alla sconfitta, se non alla morte, Segio s'interroga su quei momenti, mai veramente accessibili, in cui tutto avrebbe potuto cambiare. A questa «malinconia di sinistra nata da una

\footnotetext{
${ }^{36}$ S. SEGIO, Miccia corta, op. cit., pp. 57-59.

${ }^{37}$ Maurice BLOCH, Mémoire autobiographique et mémoire historique du passé éloigné, Enquête [In linea], 1995, v. 2, 1995, URL : http://enquete.revues.org/309 (consultato il 15 ottobre 2016).

${ }^{38}$ S. SEGIO, Miccia corta, op. cit., p. 45.

${ }^{39}$ Ibidem.

${ }^{40}$ Ibid., p. 187.

${ }^{41}$ Ibidem.
} 
sconfitta $»^{42}$ si accompagna inalienabile una fierezza, una rivendicazione del senso e degli aspetti positivi di questo stesso passato.

\section{II tempo come arma nella sfera pubblica}

Nella sfera pubblica italiana, la questione della periodizzazione del fenomeno armato sembra lungi dall'essere risolta. Negli anni, infatti, le stratificazioni del dibattito storiografico e mediatico hanno strutturato interpretazioni contrastanti fondate su scansioni temporali spesso divergenti : queste hanno a loro volta favorito la riproduzione di altrettanti crononimi - 'anni di piombo', 'stagione dei movimenti', 'anni ' 68 ', ecc. - per definire il lungo decennio Settanta ${ }^{43}$. Il nodo fondamentale di questa lunga ed articolata controversia è identificabile nel tipo di relazione - di causalità, continuità, discontinuità o differenza - che viene stabilita tra quelli che per lungo tempo sono stati riconosciuti come i fenomeni fondamentali del periodo: da un lato, il ' 68 e, dall'altro, la violenza politica e la lotta armata. Le diverse interpretazioni si sono tradotte, da un punto di vista prettamente temporale, in diverse proposte di periodizzazione : l'adozione di una scansione temporale rispetto ad un'altra sottende al tipo di rappresentazione politica e storica che si vuole trasmettere del periodo ${ }^{44}$. Anche le 'memorie armate', in quanto dispositivo culturale che permette una presa di posizione storico-politica nella sfera pubblica, partecipano a

\footnotetext{
${ }^{42}$ Enzo TRAVERSO (tr. it. Luisa CORTESE), Il secolo armato. Interpretare le violenze del novecento, Milano, Feltrinelli, 2012, p. 195 [ed. or., Paris, La Decouverte, 2011].

${ }^{43}$ Si pensi, a titolo di esempio, ai cambiamenti semantici che hanno caratterizzato l'espressione 'anni di piombo': titolo di un film di Margareth Von Trotta sul lungo 'autunno tedesco' (1981), essa viene inizialmente adottata nella sfera pubblica italiana per definire quella parte degli anni Settanta - 1976-1982 - caratterizzata da una escalation della violenza politica. In un secondo momento, però, in una rimodulazione del discorso pubblico la portata temporale dell'espressione si amplia, arrivando spesso ad inglobare tutto il decennio 70 e gli inizi dell' 80 . Cf. C. LETTIERI, «L'Italie et ses Années de plomb. Usages sociaux et significations politiques d'une dénomination temporelle », op.cit.

${ }^{44}$ In questo senso, Nando Dalla Chiesa individuava tre tipi di periodizzazione a cui accumunava tre posizionamenti politici : la 'tesi della filiazione', piuttosto di destra, tramite la quale il progetto rivoluzionario marxista espresso nel secondo biennio rosso risultava legato indissolubilmente alla deriva terroristica, vedendosi inficiata quindi ogni tipo di legittimità; la seconda tesi, quella 'dell'alterità' sarebbe caratteristica della sinistra storica e consentirebbe di isolare i due momenti riconoscendone le diversità inconciliabili, preservando quindi la legittimità del progetto marxista; la terza ed ultima tesi, quella 'dell'identità', vicina alla nuova sinistra, evidenziava un certo livello di continuità tra idee insurrezionali già presenti nel 68 e lotta armata. Nando DALLA CHIESA, « Dal sessantotto al terrorismo : cultura politica tra continuità e rottura », il Mulino, 1981, vol. 1, pp. 53-94.
} 
pieno titolo a questo dibattito. I casi presi in analisi in questo articolo permettono di esplorare diacronicamente la varietà di posizioni che contraddistinguono le varie scritture e il tipo di rapporto con il politico che esse decidono di instaurare pubblicamente. Nel caso del libro di Peci, l'aspetto genealogico e periodizzante manca quasi completamente : una prima ragione di ciò può essere ricercata nel fatto che si tratta di un testo scritto a fenomeno in corso e per intervenire più strettamente sulla sua evoluzione che sulla sua memorializzazione. In secondo luogo, questo disancoramento da una realtà e una tradizione storico-politica rivoluzionaria accentua il carattere 'privato' dell'esperienza dell'autore, insistendo sugli aspetti esistenziali ed avventurosi della sua traiettoria e minimizzando ogni considerazione sul '68 e sulla sua breve e erratica militanza in Lotta Continua. L'ammissione di questa relativa spoliticizzazione è anche un modo per attaccare i leader brigatisti che, senza alcuno scrupolo, ne approfitterebbero per i loro fini : « Il nostro livello politico, ai miei tempi, non era altissimo ma [...] quei ragazzi [di oggi] [...] non sono coscienti di ciò che fanno... Con che faccia i capi dell'Organizzazione hanno il coraggio di guardare negli occhi questi ragazzi quando se li ritrovano in galera, rovinati? $»^{45}$. In alternativa alla deriva ideologica brigatista, Peci sceglie il senso comune e la moderazione che gli fanno dire che se oggi qualcuno andasse in piazza a parlare di rivoluzione comunista irreversibile «verrebbe preso a calci nel culo e basta » e gli fanno sostenere il governo Craxi, « un buon trapasso graduale, tranquillo, senza scontri frontali $»^{46}$.

Il caso di Segio è diametralmente opposto a questo: la sua narrazione, eminentemente pubblica e politica, può essere considerata come un'operazione di differenziazione rispetto alle ricostruzioni memoriali brigatiste che, secondo l'autore, egemonizzerebbero il discorso nella sfera pubblica italiana. A questo fine, Segio mobilita differenti riferimenti storici e politici e li utilizza per costruire una costellazione di senso specifica nella quale inserire l'esperienza di Prima Linea. L'autore sintetizza questa ricostruzione genealogica in modo molto efficace : il padre di Prima Linea sarebbe il movimento del ' 77 mentre la madre, che porta in dote l'idea di violenza come motore della storia, è la concezione teorica di 'rottura rivoluzionaria'. In questo modo la paternità ancora l'esperienza nel presente del racconto mentre il lignaggio materno attribuisce una tridimensionalità storica specifica alla narrazione tramite la costruzione di una genealogia 'alternativa' dell'idea e della pratica rivoluzionarie. Se il punto di partenza di questa resta il binomio rivoluzione francese - rivoluzione bolscevica, il filo rosso della discendenza

\footnotetext{
${ }^{45}$ P. PECI, Io, l'infame, op. cit., p. 218.

${ }^{46}$ Ibid., pp. 218-220.
} 
attraversa l'esperienza anarchica e del POUM nella guerra civile spagnola, quella delle formazioni minoritarie - trotzkiste e liberal-socialiste - nella Resistenza fino ad arrivare ai movimenti sociali 'eretici' degli anni ' 70 . Si tratta di una traiettoria storica che si vuole sempre 'ai margini', in lotta col potere tout court, sia quello dello Stato che quello del dogma ideologico: una ribellione al contempo esistenziale e politica che continua anche dopo la dissoluzione dell'opzione armata. L'elaborazione della dissociazione è narrativamente resa come una maniera di continuare la tradizione critica, allergica al potere, che caratterizzerebbe la storia 'estesa' di Prima Linea. Questa alterità, però, ha un prezzo : gli accenni al dopo 1982 si concentrano sulla marginalizzazione e gli attacchi che accompagnano il ritorno di Segio e Ronconi nella sfera pubblica italiana, malgrado il loro impegno in lavori di utilità sociale. Questo aspetto diviene un'ulteriore prova del posizionamento effettivamente critico assunto da Segio durante tutta la sua storia : anche in questo caso, la strutturazione di temporalità dell'opera si presta a sostenere l'attribuzione di una veridicità - elaborata e quindi accessibile solo a posteriori - a quelle che, all'epoca in questione, non potevano essere che delle convinzioni. Per chiarire questo aspetto fondamentale, sembra pertinente fare riferimento al concetto di «memoria anticipatoria» elaborato dallo storico Andrew Bergerson ${ }^{47}$. Nella sua analisi delle corrispondenze epistolari delle classi medie tedesche durante il nazismo, Bergerson si concentra su quelle espressioni temporali intese a sormontare le incertezze del presente proiettandosi nel futuro e evocando il primo come se si trattasse già di un ricordo. Nel caso di Miccia corta, si tratta piuttosto di proiettare nel passato una certezza stabilita nel presente della scrittura e che, quel momento, non avrebbe potuto essere accessibile. Postulando la sua accessibilità, per l'appunto, l'autore può costruire una narrazione, un sistema di causalità e, di conseguenza, una rappresentazione della propria storia molto più incisive.

\section{Conclusioni}

In entrambi i casi-studio, la presa di distanza dalla strategia armata è resa narrativamente come un processo. In Peci l'arresto innesca una progressione che va dal riconoscimento 'realista' della sconfitta alla 'dissociazione', fino a giungere al 'pentimento' effettivo, inizio di una nuova vita. In Segio invece il riconoscimento della sconfitta e dell'inattualità della lotta è il primo passo del

\footnotetext{
${ }^{47}$ Andrew BERGERSON, K. Scott BAKER, Clancy MARTIN e Steve OSTOVICH, The happy burden of history: from sovereign impunity to responsible selfhood, Berlin, New York, De Gruyter, 2011.
} 
processo, a militanza ancora in corso, mentre la dissociazione, il cui racconto trova uno spazio narrativo 'spurio' nell'introduzione e nell'appendice, diventa occasione per trasporre in una cornice legittima quella lotta politica e collettiva che aveva preso erroneamente la via delle armi.

Secondo Suter, l'evento ha tre declinazioni possibili : il fatto quotidiano, l'evento e l'evento storico. Se l'evento differisce dal fatto quotidiano perché introduce una sorpresa, qualcosa di straordinario rispetto all'orizzonte quotidiano del testimone, a sua volta, l'evento storico richiede lo sguardo e il riconoscimento di una comunità più vasta che ne comprenda anche gli effetti sullo sviluppo della storia tout court ${ }^{48}$. Queste categorie, nonostante la loro staticità, rappresentano una pista interessante. Quando costruisce narrativamente la fine della sua militanza, Peci fa riferimento ad una serie di eventi che rimangono sul piano personale, non politico e non storico. La sua è una resa 'militare' articolata in due eventi : l'arresto e l'incontro con Dalla Chiesa e Caselli. In entrambi i casi, non si tratta di eventi condivisibili con il suo pubblico, in quanto non propriamente storici né particolarmente coinvolgenti sul piano emotivo, in linea con un racconto impostato come un'avventura individuale in cui le riflessioni politiche, storiche ed etiche rimangono in secondo piano. Questo aspetto è parzialmente rivisto nell'introduzione all'ultima edizione. Qui Peci condensa morte e rinascita nell'evocazione potente del momento della deposizione al processo, ricollocando così la rottura autobiografica in un setting pubblico, comprensibile e mediatizzato.

Questa strategia narrativa di individualizzazione biografica fa eco al suo comportamento giudiziario, il pentimento, che pone il rapporto tra stato e militante sul piano individuale e non politico della collaborazione e, solo eventualmente, del ravvedimento.

Il caso di Segio è più stratificato. Il suo racconto, infatti, fa riferimento ad eventi che superano la semplice biografia e che ripropongono la centralità dell'esperienza di PL, proponendo una cronologia alternativa rispetto a quella che scandisce gli anni di piombo nella sfera pubblica, imperniata sulle azioni delle BR. D'altro canto, la dissociazione per come egli la intende, gli permette di dire « se noi avevamo torto, loro [lo stato] non avevano ragione » e di rivendicare, la 'generosità' che avrebbe animato i militanti della sua organizzazione. Segio utilizza la struttura peculiare del libro per tracciare un filo rosso che, partendo dai movimenti sociali, attraversa l'evento epico e romantico di Rovigo e, passando attraverso il movimento della dissociazione, arriva fino all'oggi, 'l'altra vita' la cui storia non raccontata è rintracciabile «nelle cose che ho

\footnotetext{
${ }^{48}$ Andreas SUTER (trad. di Pierre-G MARTIN), « Histoire sociale et événements historiques. Pour une nouvelle approche », in : Annales. Histoire, Sciences Sociales. 52e année, n. 3, 1997, pp. 543-567.
} 
Mnemosyne, o la costruzione del senso $n^{\circ} 10$

detto e fatto nei successivi vent'anni, in carcere e nel volontariato, nei nuovi movimenti e nell'impegno professionale $»^{49}$.

${ }^{49}$ S. SEGIO, Miccia corta, op. cit., p. 37. 